\title{
Rhythmic Haptic Cueing for Gait Rehabilitation of People With Hemiparesis: Quantitative Gait Study
}

Theodoros Georgiou ${ }^{1 *}, \mathrm{PhD}$; Simon Holland ${ }^{2 *}, \mathrm{PhD}$; Janet van der Linden ${ }^{2 *}, \mathrm{PhD}$

\footnotetext{
${ }^{1}$ School of Mathematical \& Computer Sciences, Heriot-Watt University, Edinburgh, United Kingdom

${ }^{2}$ The Open University, Milton Keynes, United Kingdom

*all authors contributed equally
}

Corresponding Author:

Theodoros Georgiou, $\mathrm{PhD}$

School of Mathematical \& Computer Sciences

Heriot-Watt University

Edinburgh Campus

Edinburgh

United Kingdom

Phone: 441314514132

Email: t.georgiou@hw.ac.uk

\begin{abstract}
Background: Rhythm, brain, and body are closely linked. Humans can synchronize their movement to auditory rhythms in ways that can improve the regularity of movement while reducing perceived effort. However, the ability to perform rhythmic movement may be disrupted by various neurological conditions. Many such conditions impair mechanisms that control movement, such as gait, but typically without rhythmic perception being affected. This paper focuses on hemiparetic stroke, a neurological condition that affects one side of the body. Hemiparetic stroke can cause severe asymmetries in gait, leading to numerous physical problems ranging from muscle degeneration to bone fractures. Movement synchronization via entrainment to auditory metronomes is known to improve asymmetry and related gait problems; this paper presents the first systematic study of entrainment for gait rehabilitation via the haptic modality.
\end{abstract}

Objective: This paper explores the gait rehabilitation of people with hemiparesis following a stroke or brain injury, by a process of haptic entrainment to rhythmic cues. Various objective measures, such as stride length and stride time, are considered.

Methods: This study is a quantitative gait study combining temporal and spatial data on haptically cued participants with hemiparetic stroke and brain injury. We designed wearable devices to deliver the haptic rhythm, called Haptic Bracelets, which were placed on the leg near the knee. Spatial data were recorded using a Qualisys optical motion capturing system, consisting of 8 optoelectronic cameras, and 20 markers placed on anatomical lower limb landmarks and 4 additional tracking clusters placed on the right and left shank and thigh. Gait characteristics were measured before, during, and after cueing.

Results: All 11 successfully screened participants were able to synchronize their steps to a haptically presented rhythm. Specifically, 6 participants demonstrated immediate improvements regarding their temporal gait characteristics, and 3 of the 6 improved their gait in terms of spatial characteristics.

Conclusions: Considering the great variability between survivors of stroke and brain injury and the limited number of available participants in our study, there is no claim of statistical evidence that supports a formal experimental result of improved gait. However, viewing this empirical gait investigation as a set of 11 case studies, more modest empirical claims can be made. All participants were able to synchronize their steps to a haptically presented rhythm. For a substantial proportion of participants, an immediate (though not necessarily lasting) improvement of temporal gait characteristics was found during cueing. Some improvements over baseline occurred immediately after, rather than during, haptic cueing. Design issues and trade-offs are identified, and interactions between perception, sensory deficit, attention, memory, cognitive load, and haptic entrainment are noted.

(JMIR Biomed Eng 2020;5(1):e18649) doi: 10.2196/18649 


\section{KEYWORDS}

hemiparetic gait; stroke; technology assisted rehabilitation; quantitative study; gait analysis; gait asymmetry; gait; neurology; hemiparesis; rehabilitation; brain injury

\section{Introduction}

Brain injury following an accident or stroke can leave people with life-changing neurological conditions and a general weakness on one side of the body. Motor control of one side of the body can be severely affected with unilateral loss in sensation and muscle coordination of both upper and lower limbs.

Motor control deficiencies can lead to spatial and temporal asymmetries affecting walking in a condition known as hemiparetic gait. The asymmetries can cause sufferers of hemiparetic gait to overuse their nonaffected (nonparetic) leg, exposing it to potentially damaging, higher vertical forces [1,2], while underuse of the paretic (affected) leg can lead to loss of muscle tone and reduction of bone mineral density [3]. These effects, in turn, increase the risk of knee and joint problems, hip and bone fractures, and falls [4].

Walking following an external metronomic rhythm has been shown to improve gait, leading survivors of stroke to walk more symmetrically [5] and to neglect their affected leg less.

Early studies by Prassas et al [6] using rhythmic auditory stimulation (RAS) - a neurological technique using audio rhythms for facilitating rehabilitation, development, and maintenance of movements that are fundamentally biologically rhythmical-have shown immediate spatial benefits, with stride lengths becoming more symmetrical. In addition, the work of Prassas et al [6] found the hip joint range of motion to increase, and the center of mass displacement to decrease, during walking with RAS, making the overall forward movement smoother.

More recent studies found that survivors of stroke could easily synchronize their steps to a rhythmic audio metronome while walking on a treadmill; this led to improvements in various temporal symmetries, including improved step time symmetry between the paretic and the non-paretic leg [7], as well as reduced variability in the paretic step times [8].

These results indicate that RAS and, by extension, walking to a rhythm has clear clinical significance since step asymmetry is a leading cause of many problems associated with neurological conditions like hemiparetic gait [9]. More specifically, improvements in symmetry and variability suggest steps toward functional recovery of gait mechanics, as they are kinematically associated with a healthy gait [9].

However, auditory cues can be impractical to use outside of the clinic, or to use in situations where it is desirable to maintain environmental awareness or social engagement. Moreover, the way in which audio cues are presented to patients during rehabilitation in the clinic introduces particular limitations. In order to maintain communication between patients and physiotherapists, audio cues are usually played out through speakers. In addition, for optimum entrainment results, the tempo must match the patient's walking pace [10]. Therefore, only one patient can receive the audio cues at a time, confining the number of sessions that can run simultaneously in the same space to one. This could be problematic in situations where the same space (ie, a gym inside a rehabilitation clinic) is shared by more than one physiotherapist.

Haptic rhythms, on the other hand, can be directed to more than one patient simultaneously, each with their own tempo to match their cadence, without interfering with each other. This enables more efficient sharing of resources between health professionals and physiotherapists.

Cueing of the steps of each leg has demonstrated stronger auditory-motor synchronization than cueing those of just one leg (either paretic or nonparetic) [10]. However, interpreting an audio rhythm that differentiates cues for the paretic and nonparetic leg can challenge those needing concentration to walk at all.

A pilot study attempting to assign cues of different pitches to each leg [8] identified a number of limitations to this approach, mainly an illusion created by the pitch differences between successive cues causing an isochronous (regular) rhythm to be perceived as irregular. In addition to this acoustic illusion, participants in the same pilot study reported that having a rhythm of two tones is difficult to understand, with one participant withdrawing from the study because they "did not like the dual-tone" [8].

The haptic sense, on the other hand, has the potential to focus attention and proprioception on each leg by simply applying a tactile pulse to each leg in turn, in a more embodied, directly spatial, and less burdensome way.

As a systematic extension of an earlier pilot [11], this study is designed to investigate the effects of rhythmic haptic cueing on diverse gait characteristics associated with healthy kinematics and gait patterns. These include spatial characteristics (ie, stride lengths), temporal characteristics (ie, step, stand, and swing times), and derivative asymmetries, calculated from the spatiotemporal characteristics.

\section{Methods}

\section{Procedure}

This was a single-session, repeated-measures study. Participants with hemiparetic brain injury were first asked to walk a short distance without intervention to establish a baseline, and then their pace was matched to an isochronous (regular) haptic rhythm whose period matched a symmetric version of their natural cadence, calculated by averaging the time in milliseconds between each successive step. Subsequently, any resulting residual effects (such as rhythm persistence and the ability to walk to the rhythm from memory) were tested by asking the participants to walk to the rhythm from memory shortly afterward.

The default placement of the wearable devices we designed to deliver the haptic rhythm, called Haptic Bracelets, was on the 
leg near the knee. This area, chosen due to findings from an earlier pilot study [12], is covered in what is termed "hairy skin," which has a low concentration of the particular mechanoreceptors (sensory neurons) responsible for perceiving vibrational stimuli, namely Pacinian and Meissner's corpuscles. Hairy skin does, however, contain Merkel's disk and Ruffini endings, which can detect pressure and skin stretch [13]. Generally, tactile cues coming from the vibrotactile actuators of our devices are perceived more as a tap sensation than as a vibration, as reported by several participants in pilot testing [12]. This suggests that the dominant mode of perception will not be vibrational.

During this study, when choosing the intensity of cues, an informal qualitative approach was preferred, ensuring firstly that the participants could feel the cues, and then adjusting the intensity to personal preference. Author TG [14] engages in a full discussion of the technical implementation of the devices, the engineering of the haptic cues, special considerations made to enhance the tap sensation of each cue, and lab testing to refine the device and its placement in Chapter 5 of his work "Rhythmic Haptic Cueing for Gait Rehabilitation of Hemiparetic Stroke and Brain Injury Survivors."

In order to identify quantitative changes in gait, two kinds of data were collected, involving temporal and spatial asymmetry, respectively; these measures often play a role in guiding the clinician's treatment decisions [15]. This involved measures of paretic and nonparetic step timings and measures of paretic and nonparetic step length.

Temporal data were collected from all participants using the Haptic Bracelets wearable devices. The Haptic Bracelets are part of a wearable prototype system designed and developed at the Open University for the purpose of gait monitoring and gait rehabilitation through rhythmic haptic cueing [14].

Haptic Bracelets work in pairs (one for each leg). Each Bracelet contains sensors integrated in an inertia monitoring unit (IMU) for recording appropriate motion data. This data is used for calculating gait characteristics, including gait asymmetries and step variability in terms of overall temporal asymmetry (OTA) ratio values. Each unit also has a vibrotactile actuator capable of producing a tactile cue of comfortable quality but sufficient intensity to be felt by the user. Having one unit on each leg creates a multi-limb tactile metronome, producing tactile cues on alternating legs. This allows for the cueing of each limb independently, facilitating the investigation of effects related to attention and proprioception in the context of gait rehabilitation. In addition, this multi-limb approach has the additional advantage of motion data being gathered from each leg independently. This makes it possible to easily and clearly distinguish between the motion of the paretic leg (affected by the neurological condition) and the nonparetic leg. This improves the accuracy of the measurement of temporal characteristics and the analysis of asymmetry.

Temporal data was collected for all 11 participants (easy anywhere using the Haptic Bracelets). However, due to limitations in the geographical availability of participants, the collection of spatial data was limited to just 6 participants, as this was possible only at an optical motion capture facility that was local to these 6 participants. Figure 1 shows the lab setup during this study.

Figure 1. Participants during a 10-meter walking trial. The photograph on the right was taken during a trial in the optical motion capture lab. The photographs on the left and the right feature separate rooms; however, both locations offered an identical setup and procedures for this part of the study.
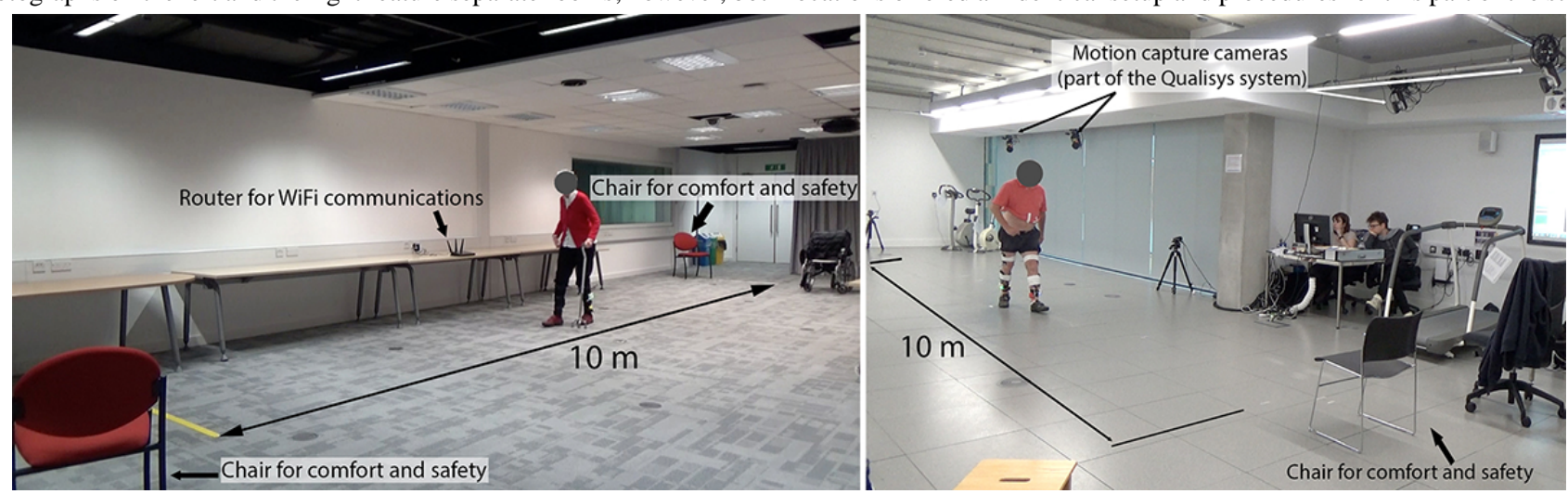

\section{Participants}

The participants in this study were 11 community-dwelling, community-ambulant adults ( 7 men and 4 women) with chronic hemiparesis ("chronic" defined as >6 months since stroke onset). For 10 of the 11 participants, hemiparesis followed a stroke; for the other participant, it was due to brain trauma following an accident. The age range of the participants is shown in Table 1 , and the time since the occurrence of stroke varied from 8 months to 12 years. All participants gave written, informed consent to participate.
Participants were recruited through local support groups and the recommendations of private physiotherapists. Health professionals collaborating with our team for this study were responsible for determining the conditions of all of the inclusion and exclusion criteria. Inclusion criteria were (1) walking disability, but with a retained or subsequently recovered ability to stand and ambulate; (2) the ability to walk unsupported (but with a walking aid if needed) for a minimum distance of 10 meters; (3) a Rivermead Motor Assessment (RMA) scale score of more than 8 . 
Table 1. Participant demographic information $(n=11)$.

\begin{tabular}{ll}
\hline Participant characteristics & Values \\
\hline Age in years, mean (SD) & $61.75(7.85)$ \\
Gender, $\mathbf{n}$ & 7 \\
$\quad$ Men & 4 \\
$\quad$ Women & \\
Paretic side, $\mathbf{n}$ & 9 \\
$\quad$ Right side & 2 \\
$\quad$ Left side & 2 \\
\hline
\end{tabular}

RMA is a standard and widely used test for assessing functional mobility in gait, balance, and transfers after stroke [16]. A score of 8 and higher was selected as the inclusion criterion following discussions with physiotherapists. RMA scores were collected in private by the health professionals (private physiotherapists and other health professionals working for support groups) handling our recruitment.

Participants were excluded if they had cognitive impairments preventing understanding of the task. Participants could use their assistive devices (ankle-foot orthosis splint or cane) in the trials.

\section{Prestudy Preparation}

For the contingent reasons noted above, the study took place in two locations: the 6 local participants attended a kinematics lab in Manchester with an optical motion tracking system, and the 5 remaining participants attended a large observation lab in Milton Keyne. All participants had temporal data collected, but only those attending the optical tracking lab (6 out of the 11) had spatial data collected simultaneously (Table 2). An identical procedure was followed in both locations, except that 3 additional preparatory steps were required to meet the needs of the optical motion tracking system. Namely, participants at the kinematics lab location were asked to change into shorts, 30 to 45 additional minutes were required for a trained technician to place all of the optical tracking markers on their body (Figure 2), and biometric measurements were taken (ie, height and weight).

Table 2. Demographic data for each participant per location. Participants with an MMU code took the study at the Manchester location; those with an OU code took the study at Milton Keynes.

\begin{tabular}{ccclll}
\hline $\begin{array}{l}\text { Participant code \& loca- } \\
\text { tions }\end{array}$ & $\begin{array}{l}\text { Age in } \\
\text { years }\end{array}$ & Gender (M/F) & Years since brain trauma & Paretic side (Left/Right) & Data collected (Spatial/Temporal) \\
\hline \multicolumn{2}{l}{ Manchester (kinematics lab) } & 53 & F & 12 & Right \\
MMUP01 & 57 & F & $0(8$ months) & Right & Spatial and Temporal \\
MMUP02 & 73 & M & 2 & Left & Spatial and Temporal \\
MMUP03 & 68 & M & 7 & Right & Spatial and Temporal \\
MMUP04 & 61 & M & 5 & Right & Spatial and Temporal \\
MMUP05 & 55 & F & 4 & Right & Spatial and Temporal \\
MMUP06 & 50 & M & 21 & Spatial and Temporal \\
Milton Keynes (observation lab) & & 9 & Right & Temporal only \\
OUP01 & 50 & M & 9 & Right & Temporal only \\
OUP02 & 60 & M & 2 & Right & Temporal only \\
OUP03 & 73 & F & 1 & Right & Temporal only \\
OUP04 & 56 & M & 1 & Left & Temporal only \\
OUP05 & & & &
\end{tabular}


Figure 2. Left: Haptic Bracelets wearable devices (metronome and monitoring units) and vibrotactiles strapped on a participant's leg using Velcro straps. Right: all the optical markers attached to a participant's body.

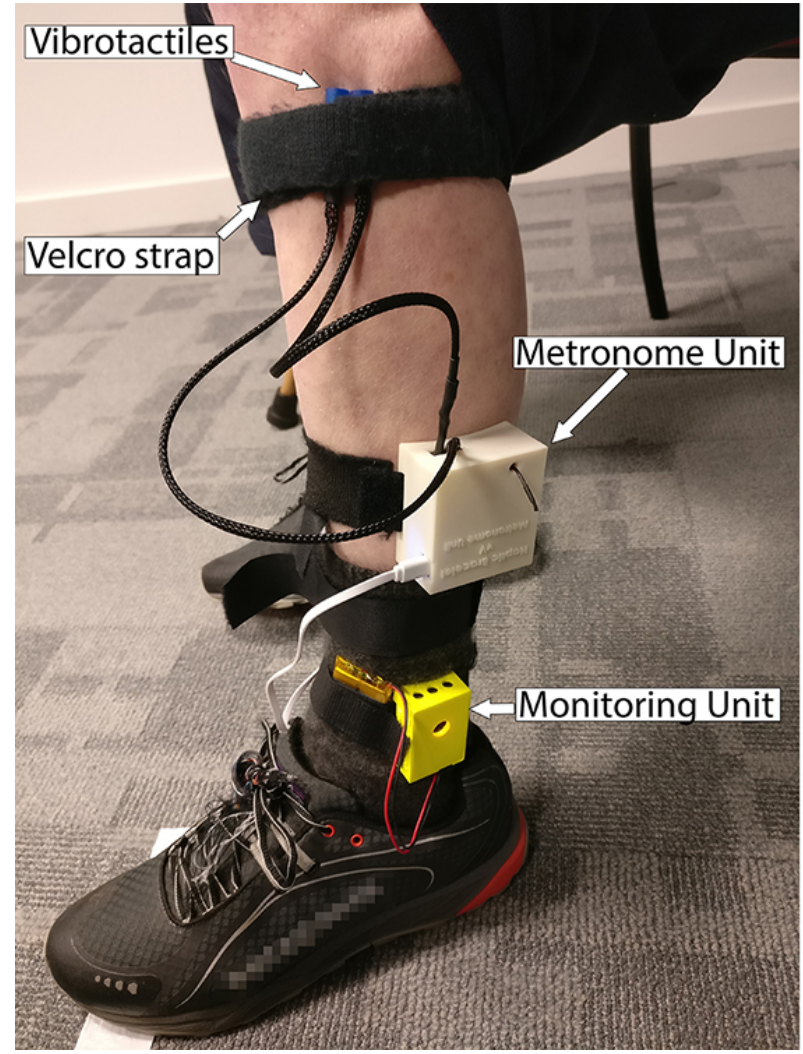

In both locations, the Haptic Bracelets were attached via Velcro straps onto the tibia of each leg near the ankle. The vibrotactile (the part of the device that gives the haptic cue) was attached using another Velcro strap near the knee (Figure 2).

The placement of the vibrotactile was initially based on the suggestion of physiotherapists participating in an earlier study [12]. However, this decision was later revealed to be based on a conflation by the physiotherapists of stimulus response with entrainment (ie, conflating functional electrical stimulation aimed at a direct muscle response with neural entrainment, where a regular external pulse leads to a matching, predictive internal neural resonance [12]). Nonetheless, it was subsequently decided to maintain this placement for this study for two main reasons: (1) proximity to major nerves, giving the tactile cue a good chance to be felt; and (2) having the vibrotactile unit away from the IMU of the Haptic Bracelet helps to minimize unwanted noise in the gait data.

Participants who did not have their spatial data monitored had a simpler preparation than those that did; they did not have to wear any kinematics markers and could simply wear a pair of Haptic Bracelets over or under their everyday clothes (depending on preference).

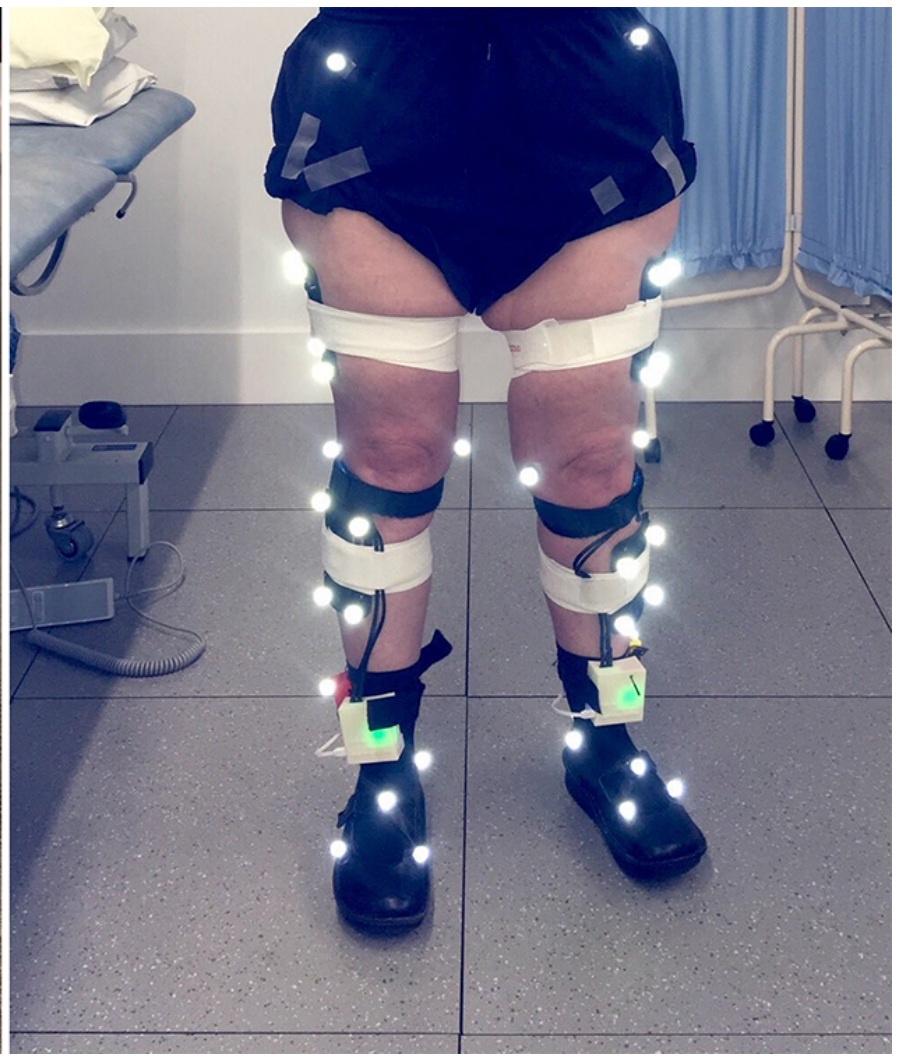

The Haptic Tap Test

Before the first trial, all the participants were asked to take a haptic rhythm perception test, called the haptic tap test. Performance in this tap test was not used as an inclusion criterion for the study but was rather used to investigate the participants' rhythm perception capabilities.

During rhythm-based rehabilitation sessions, it is not easy to distinguish between survivors of stroke who simply have physical difficulties walking to a rhythm from participants who are unable to entrain to a rhythm for other perceptual or cognitive reasons. This ability to perceive a rhythm can be affected by injury to certain parts of the brain [17]. Such a deficit may go undetected by medical professionals and is not identified by standardized gait assessment tests such as the Rivermead Mobility Index [16] and the timed-up-and-go (TUG) test [18].

Therefore, we administered a rhythm perception test. In this test, patients tap a button following a steady rhythm presented to them haptically via a vibrating unit fixed on their wrist (Figure 3 ). Their ability to synchronize their tap tempo to the rhythm can be used for assessing their ability to perceive and follow, or entrain to, the given rhythm. Consequently, this test can be used for identifying patients who are (1) able to entrain to rhythms and (2) not impaired in the ability to entrain to rhythm presented haptically. 
Figure 3. Participant performing a haptic tap test by tapping a button with his right index finger. The vibrotactile of the Haptic Bracelet device is secured on his left wrist using a pair of Velcro straps.

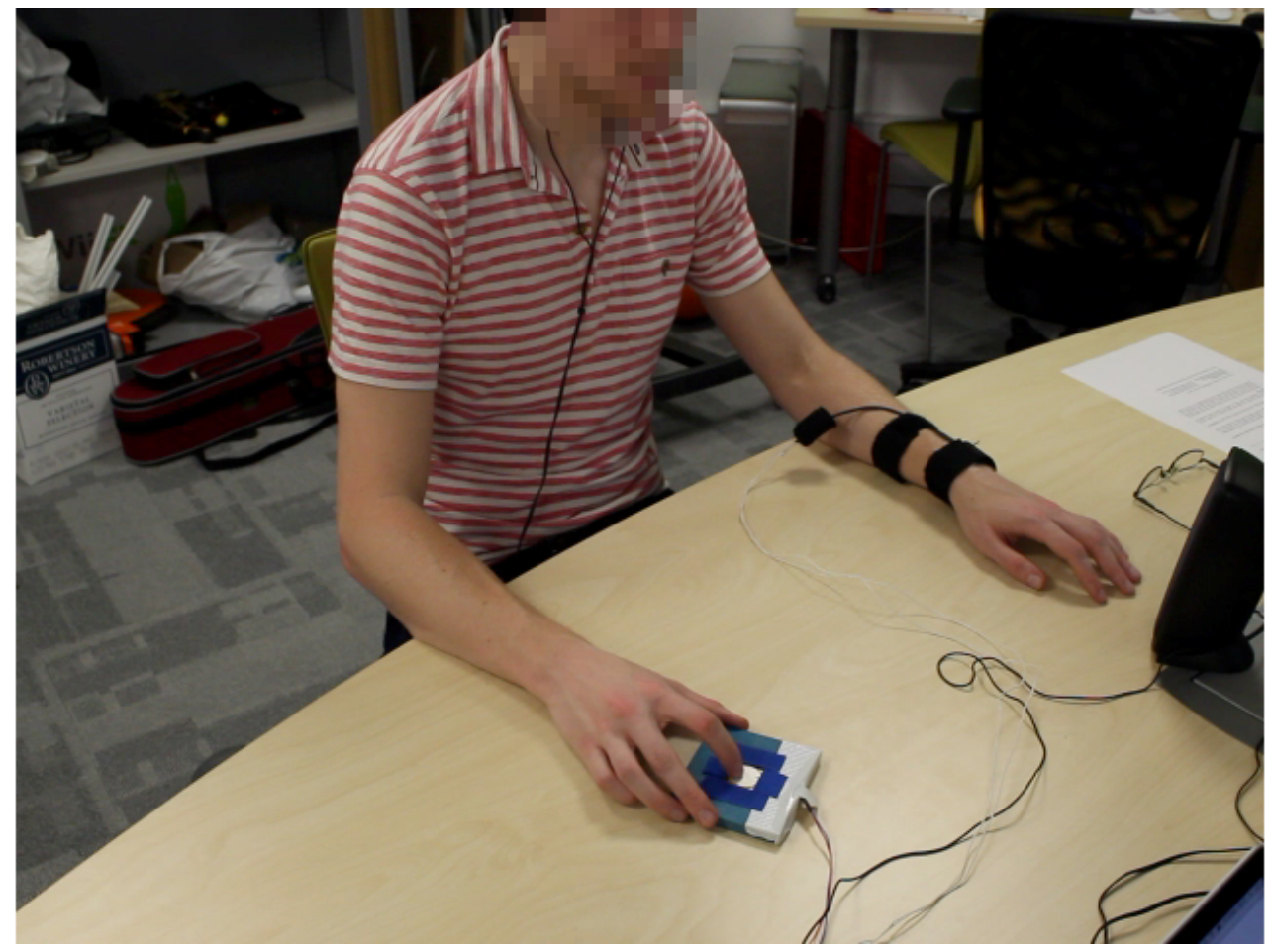

The haptic tap test allows for an empirical indication of the participants' cognitive and perceptual abilities to entrain to a rhythm for movement rehabilitation, aiming to assess people's ability to perceive and replicate a rhythm (albeit by tapping) perceived via the haptic modality.

To conduct the haptic tap test, participants were asked to tap with their index finger on a sensor in time to a range of rhythms. The rhythm was delivered haptically on the paretic wrist, using a wired version of the Haptic Bracelets. Tap times were recorded digitally. Tapping was performed by the nonparetic hand in order to avoid any physical constraints due to hemiparesis or the effects of haptic masking [19]. Haptic masking describes situations where haptic sensation is temporarily muted or attenuated by adjoining muscle movement. Participants were all tested with rhythmic periods of 500, 600, and $700 \mathrm{~ms}$. Based on experiences from pilot tests and in a previous, similar study involving able-bodied participants, each trial lasted just 20 seconds to minimize participant fatigue. The ability to tap was observed visually during the task and was also analyzed from the digital data log after the conclusion of each session. Flawless performance was not required; a general ability to keep in time with the beat was sufficient to pass the test.

\section{Walking to the Rhythm Trials}

This study followed a repeated-measures design with three conditions: baseline, cued, and post. The participants were asked to walk the length of a 10-meter runway 6 times for each condition. A 5-minute break was offered between each condition.

\section{Baseline Condition}

The initial condition allowed each participant's baseline gait to be measured, including mean step time. The mean step time was used to set the period of the haptic metronome for the subsequent cued condition, subject to any final adjustments.

\section{Choosing the Period for the Cued Condition}

This approach to choosing the cueing period was motivated by the literature, but with scope for practical adjustments to cope with the realities and comfort of hemiparetic participants. One of the underlying neurological principles defined in the RAS rehabilitation technique is to entrain steps to an external rhythm that, as nearly as possible, matches the preferred (uncued) cadence of the patient [20]. Independently, Roerdink [10], working with elderly able-bodied participants, found optimal performance when cueing with a regular period close to each individual's naturally preferred cadence. Cues in this study were delivered to alternate legs, with evidence from auditory cueing emphasizing the benefits of this approach [21].

Bearing in mind the characteristic temporal asymmetry of hemiparetic gait, the average period, calculated from a hemiparetic individual's preferred pace, may be too fast for one leg and too slow for the other; this suggests that some trial-and-error adjustments of the period may be desirable. Also, some participants needed to apply conscious effort to walk naturally, and thus, some might feel less confident when asked to undertake an additional task while walking. For these two reasons, some leeway in the preferred adjustment of the period was allowed.

\section{Familiarization Period}

In order to find a comfortable period, close to the preferred period before commencing with cued walking, participants were variously asked to tap their foot while sitting down, or to step in place while standing up, or to walk around following the rhythm, as was most convenient for them. This familiarization period allowed final adjustments to the period and to the 
intensity of the haptic cue. The haptic cue intensity was adjusted to meet each participant's personal preference, making sure they could feel it while walking rather than trying to home in on specific frequencies for mechanoreceptor activation.

Findings from an earlier study using a technology-probe approach [12] highlighted the importance of clear instructions on entraining steps to a rhythm. Participants were instructed to time the steps of their nonparetic legs to the beat but not to worry about the cadence of their paretic leg. They were encouraged to feel the rhythm in a similar manner as one would to a song. After testing various instructions through trial and error during the earlier pilot study [12] and with pilot volunteers in the lab, this instruction was found to generally lead to a more balanced gait than asking participants explicitly to time the steps of both legs to their respective beats.

Here an interesting issue arises. One might be tempted to engineer a cue where the ratio of step periods between legs lay at some intermediate point between the participant's baseline asymmetry and a perfectly balanced symmetry. However, a wide range of studies [22-25] indicate that rhythms with exactly regular periodicity are best at engaging with human neural mechanisms for entrainment to allow upcoming beats to be precisely predictable, thus facilitating bodily entrainment. Similar considerations apply when recalling previously heard rhythms [14,22,24]. These findings agree with observations during in-lab pilot testing, where participants generally found it much easier to entrain to a regular beat than an irregular or "swung" beat (in musical terminology).

\section{Cued Condition}

Once participants confirmed that they understood the instructions, they were asked to walk six 10-meter lengths following the haptic rhythm. Temporal data and, in the case of the participants in the Manchester lab, spatial data were recorded for each walk. A short 5-minute break was offered between the cued and post conditions.

\section{Post Condition}

Immediately following the cued condition, and after a short 5-minute break, each participant was asked to repeat a further six 10-meter walks without haptic cueing. The purpose of this post condition was to investigate any residual effects of walking to the rhythm. This was inspired by literature on Parkinson disease reporting rhythm persistence [26-28], and also by comments from a participant in the pilot study [12] who noted how the rhythm remained in their memory; "If it is switched off [...], it's still there. [...] in my head." (Participant 4 [12]).

In addition to a short 5-minute break scheduled between conditions, participants were told they could take a break at any point during the study. The study concluded with a short, unstructured discussion about the participant's experience walking with the haptic rhythm to help us understand how our gait rehabilitation method was perceived and if any methodological or technological improvements or alterations were necessary. All trials were video-recorded for later review by expert physiotherapists as, even if they were present in the room during the cued and post conditions, they were often concentrating more upon the participant's safety than subtle changes in gait pattern and body movement.

\section{Data Analysis}

When assessing gait, it is useful to break walking down into various constituent elements. For each step, the foot lifts off the ground, swings forward, hits the ground, and stands until ready to lift off again, forming one step cycle (Figure 4).

Figure 4. Phases in human gait. The swing phase is defined from the moment the toes of the foot initiating the step lift off the ground and the leg begins to swing forward. This phase completes when the heel of that foot strikes the ground, starting the stance phase. Between two successive step cycles of alternating legs, there is the double support phase, where both legs touch the ground (the time between the heel strike of one foot and toes off of the other foot). This is an integral part of walking; however, double stance time does not affect how gait symmetries are calculated in this paper.

\section{Human Gait}

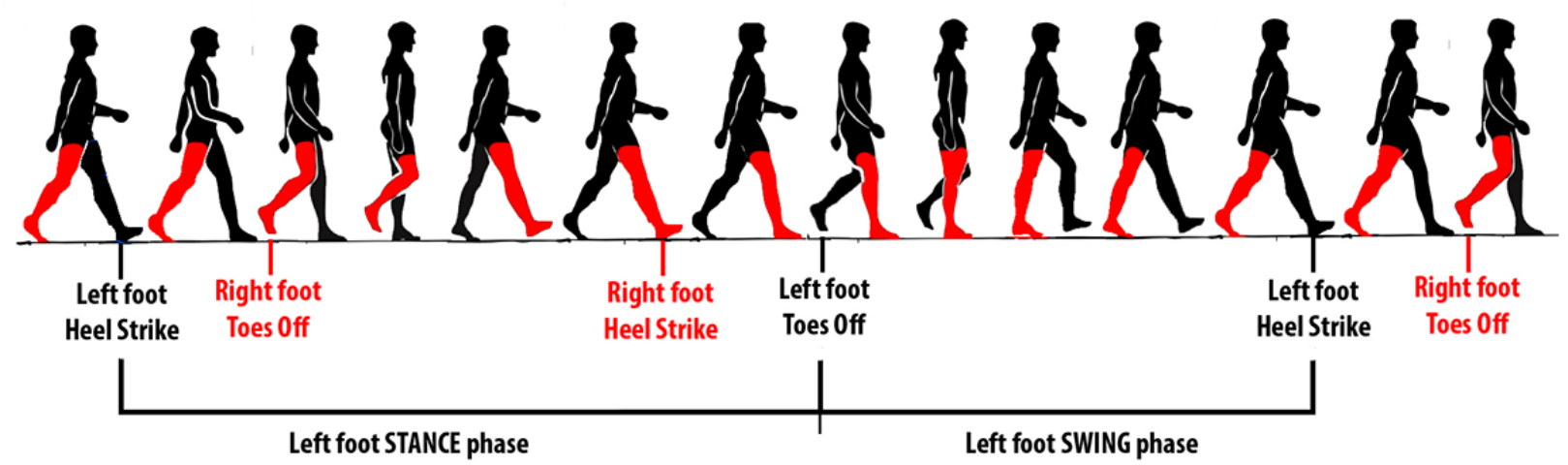

One Left foot step cycle

One Left foot step 
Multiple-step cycles happen for each alternating leg. Therefore, each leg acts as an oscillator performing a fundamentally cyclic process on every step. However, unlike a simple pendulum, the cycle time of normal gait is not purely mechanically determined and can exhibit any of a wide range of periods. Gait patterns can be entrained to a suitable external rhythm by a neurologically mediated process, as described in the following summary adapted from Holland et al [29]. The concept of entrainment, originally from physics, describes how two or more physically connected rhythmic processes can interact with each other to adjust towards, and eventually lock into, a common or closely related periodicity and phase. However, the concept has unexpected application in areas as diverse as perception, cognition, and music. The best current account of the underlying brain mechanism comes from Neural Resonance Theory [22,23]; it proposes that humans have a specialized neural organ consisting of a bank of actively powered oscillators with temporal periods from about $200 \mathrm{~ms}$ to 2 seconds. Human entrainment can be explained by the way in which these hypothesized oscillators tend to entrain with sensory input and are able to entrain, in turn, with motor processes such as gait. Given that rhythm and gait rehabilitation are focal points of this study, the capacity of gait to entrain with external rhythms is of vital importance.

In this study, temporal and spatial data were recorded and analyzed. Temporal data refers to times between events (eg, time between subsequent heel strikes) and other gait characteristics that can be calculated from these timings. In particular, temporal data were analyzed and assessed using the OTA ratio.

\section{Overall Temporal Asymmetry (OTA) Ratio}

First, the swing-stance ratio is calculated for each leg (1). This is the ratio (1) between the swing (SW) and stance time (ST) for each leg in turn.

$$
\text { Swing } / \text { stance ratio }=\frac{S W}{S T}
$$

The OTA ratio (2) compares the swing-stance ratio of the paretic leg with the nonparetic leg.

$$
\text { Overall temporal asymmetry }=\frac{s W_{p}}{s T_{p}} / \frac{s W_{n p}}{s T_{n p}}
$$

For a healthy individual, the value for the OTA should be between 0.9 and 1.1, which is a range described as healthy or normative [15]. In the case of neurological conditions, higher swing-stance ratios are commonly seen. For example, in the case of hemiparesis, one side of the body is affected unilaterally, typically causing the affected leg to swing more slowly. Due to weakness and sensitivity loss, a survivor of stroke often loses trust in the affected leg, doubting it can support them. This further causes them to swing the nonaffected (nonparetic) leg faster in order to minimize the time spent standing on the affected leg. Consequently, the swing time of the nonparetic leg decreases (ie, swings faster), causing the stance time of the paretic leg to also decrease.

These combined changes in stance and swing times of both legs raise the OTA value. Generally, while 0.9 to 1.1 describes normal asymmetry, values between 1.1 to 1.5 describe mild asymmetry, while values over 1.5 describe severe asymmetry [15]. As previously noted, entraining to an external rhythm has been shown to reduce gait asymmetry and results in a more symmetric and less variable gait pattern [24].

\section{Spatial Data Analysis}

Spatial data are used to determine gait characteristics relevant to space, such as stride length. Spatial data were recorded using a Qualisys optical motion capturing system. The system consists of 8 optoelectronic cameras, with a sampling frequency of $100 \mathrm{~Hz}$ [30]. The trajectories of 20 markers placed on anatomical lower limb landmarks and 4 additional tracking clusters placed on the right and left shank and thigh (Figure 2) were collected and filtered using a fourth-order, zero-lag, low-pass Butterworth filter with a $6 \mathrm{~Hz}$ cut off.

Each marker is tracked by the cameras, triangulating its position in space. This allows for the tracking of motion in 3 degrees of freedom, to millimeter accuracy. For spatial data, the movement of the markers in the forward direction was calculated for each step.

Spatial and temporal data collected during the cued and post conditions were compared against the baseline, looking for any effects that entrainment to haptic rhythmic cueing might have on the participant's gait. Comparing the data against the baseline allowed each participant to act as their own control, making clear any walking effects that were caused by the cue.

\section{Results}

\section{Outliers}

All data were examined for outliers. Outlier values were identified using the Tukey fence method. The Tukey fence method defines outliers as any values lying at a distance greater than 1.5 times the interquartile range. For characteristics that jointly contribute to the step time (ie, swing and stance times), if either value was considered to be an outlier, both values were removed from the analysis.

\section{Temporal Data}

As previously discussed, paretic and nonparetic step timings were determined from initial footfall contact by the Haptic Bracelet's onboard sensors. Analyzing data from different sensors and combining the information together allowed for the analysis of stance and swing times. From these, the OTA ratio value was calculated for every participant in every condition (baseline, cued, and post).

The average reduction on the OTA values across all participants indicates an overall improvement, with the OTA value approaching normative asymmetry levels (normative range: 0.9-1.1 [15]). The results are summarized in Figure 5. 
Figure 5. Mean overall temporal asymmetry (OTA) values from all participants for all three conditions: baseline, cued, and post. The figure includes normative levels indicating healthy walking symmetry. Error bars show 1 standard error of mean.

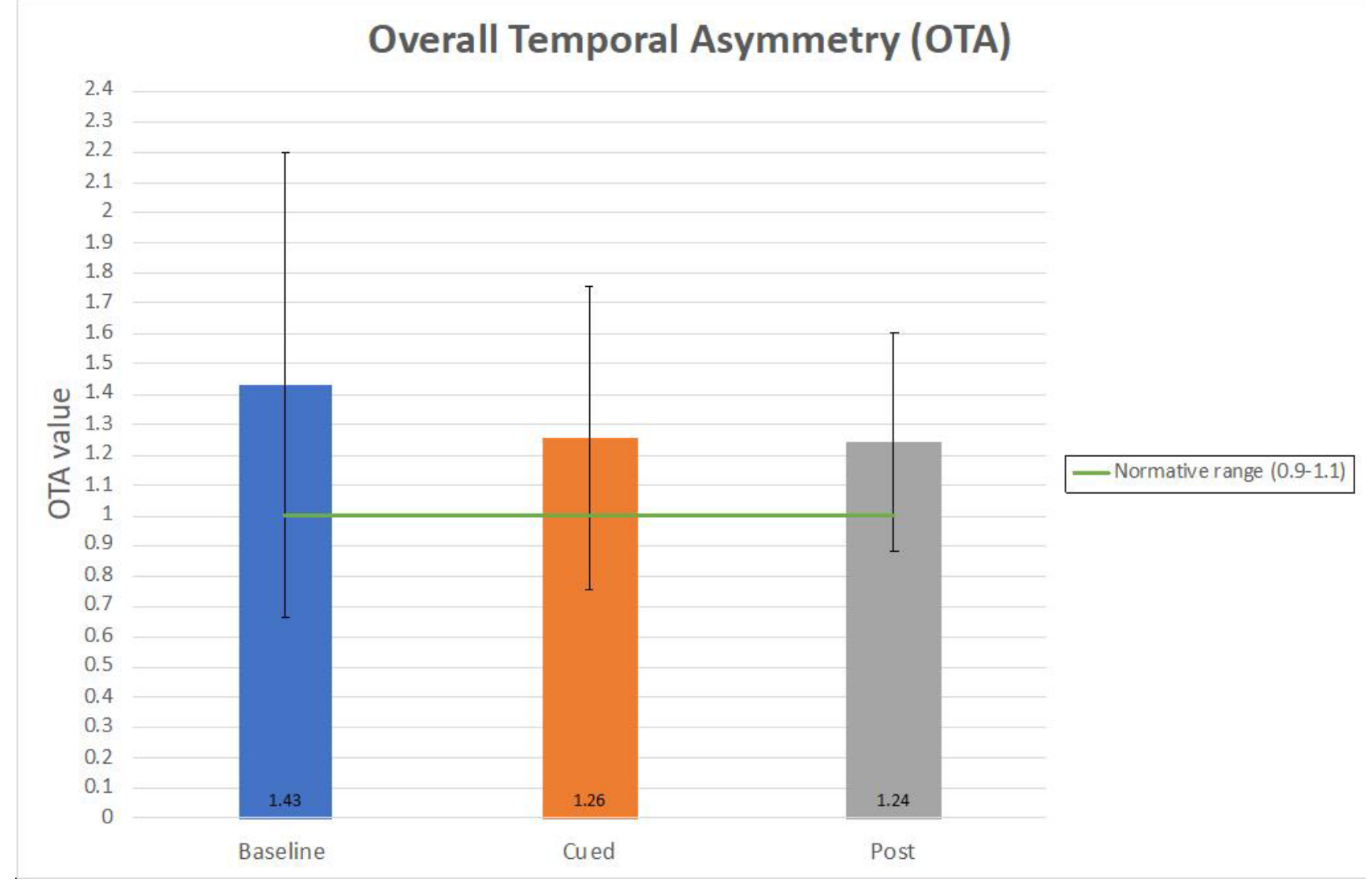

\section{Methodological Note: Comparing Baseline, Cued, and Post Conditions}

Due to the high degree of variability between survivors of hemiparetic stroke (Figure 5) and the relatively small number of participants, statistics only reveal a limited part of the story; more information can be gleaned from the details of individual cases.

\section{Consideration of Individual Results}

Due to the inherently wide variability between individual survivors of stroke and brain injury, changes to the OTA value were normalized by calculating the percentage changes from each individual's baseline value. Negative percentage values indicate beneficial change (ie, approach the normative value range of 0.9 to 1.1). These data are shown in detail in Table 3, graphed in Figure 6, and summarized for clarity in Figure 7. 
Table 3. The overall temporal asymmetry (OTA) values of all participants in all three conditions (baseline, cued, and post; $\mathrm{n}=11$ ).

\begin{tabular}{|c|c|c|c|c|c|}
\hline \multirow[t]{2}{*}{ Participant code } & \multicolumn{3}{|l|}{ OTA } & \multicolumn{2}{|c|}{ OTA percentage change from baseline $(\%)^{\mathrm{a}}$} \\
\hline & Baseline & Cued & Post & Cued & Post \\
\hline MMUP01 & 1.32 & 1.23 & 1.51 & $-6.99^{b}$ & 14.37 \\
\hline MMUP02 & 1.08 & 1.06 & 1.04 & $-1.94^{b}$ & $-3.89^{b}$ \\
\hline MMUP03 & 2.27 & 2.46 & 1.83 & 8.46 & $-19.16^{b}$ \\
\hline MMUP04 & 1.11 & 1.04 & 1.25 & $-6.66^{\mathrm{b}}$ & 12.30 \\
\hline MMUP05 & 1.84 & 1.58 & 1.64 & $-14.11^{b}$ & $-10.88^{b}$ \\
\hline MMUP06 & 1.16 & 1.07 & 1.22 & $-7.36^{\mathrm{b}}$ & 5.13 \\
\hline OUP01 & 0.85 & 0.92 & 0.88 & $-7.72^{b}$ & $-3.06^{b}$ \\
\hline OUP02 & 1.14 & 1.14 & 1.18 & 0.44 & 3.80 \\
\hline OUP03 & 3.32 & 1.75 & 1.56 & $-47.35^{b}$ & $-52.93^{b}$ \\
\hline OUP04 & 0.79 & 0.79 & 0.80 & $-0.04^{b}$ & 0.17 \\
\hline OUP05 & 0.86 & 0.76 & 0.74 & 11.68 & 14.01 \\
\hline
\end{tabular}

${ }^{\mathrm{a}}$ Represents the change from the baseline toward the normative range of symmetry $(0.9-1.1)$, representing the healthy portion of the population.

${ }^{\mathrm{b}}$ Negative values indicate an improvement in symmetry.

Figure 6. Graphical representation of OTA values shown in Table 3. Perfect symmetry (1.0) and normative asymmetry levels in the range of 0.9-1.1 are shown by horizontal green lines.

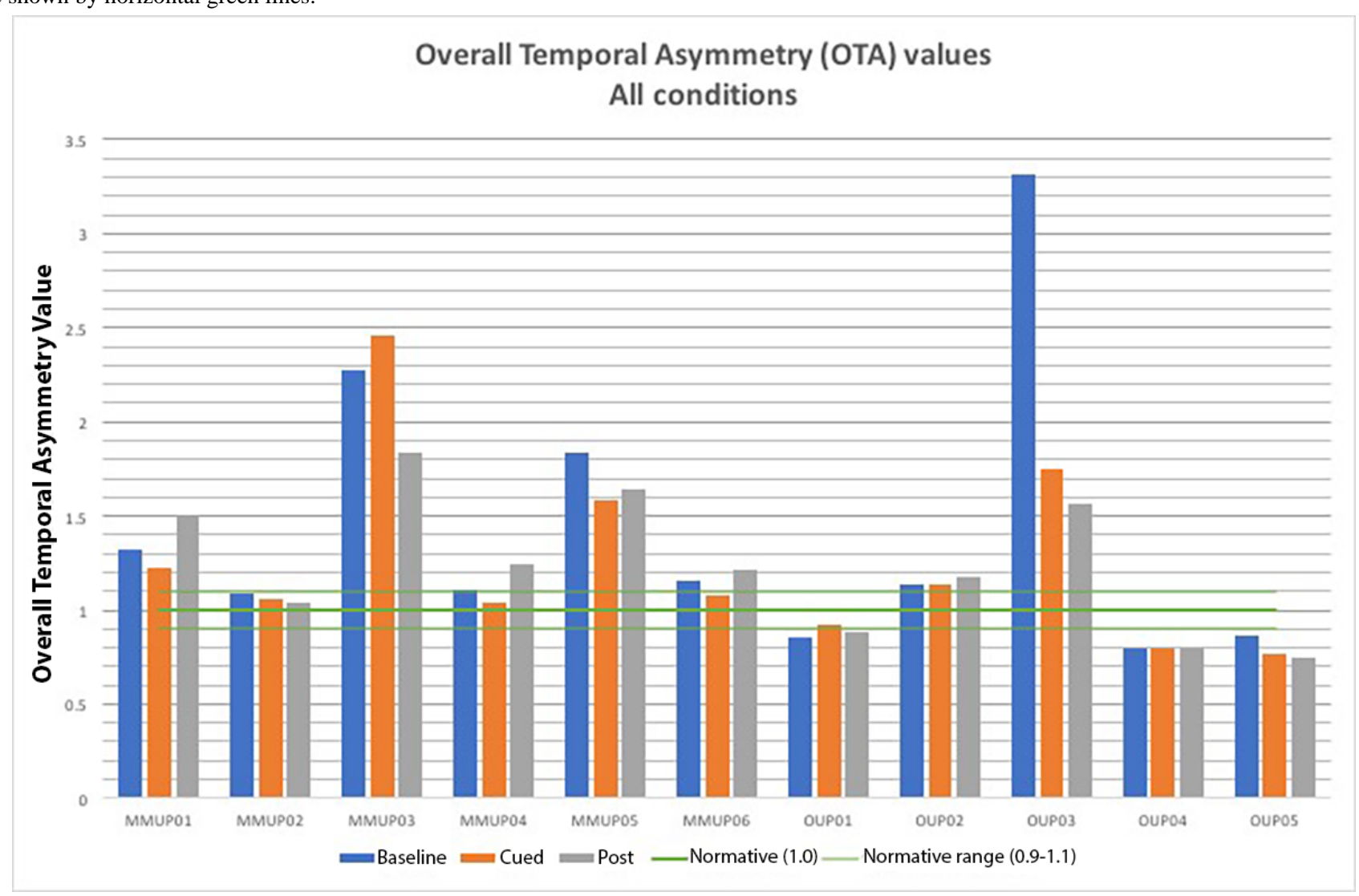


Figure 7. Temporal changes. The participant distribution is based on their overall-temporal-asymmetry percentage change compared to baseline. The three study conditions (baseline, cued, and post) are represented by the three levels of the flowchart.

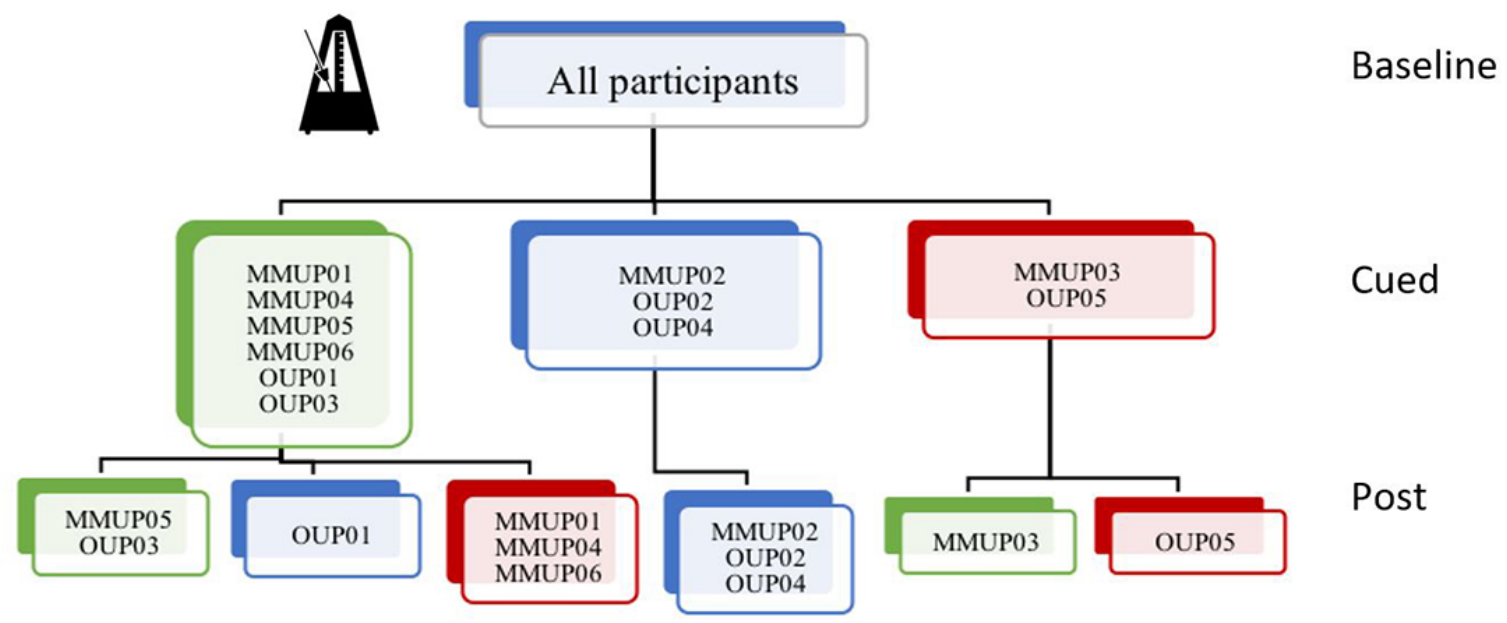

\section{: symmetry improvement* $\square:$ symmetry decline* $\mathbf{0}$ : no change*}

\section{*compared to the baseline of each individual}

\section{Temporal Data Results Summary}

In this study, three outcomes were possible for the cued and post conditions: Compared to the baseline, a participant's OTA could improve, worsen, or stay the same. Alterations of the OTA were considered meaningful if their magnitude was $\pm 5 \%$ compared to each individual's baseline.

As shown in Table 3 and Figure 6, six of the 11 participants with brain injury exhibited immediate improvements in their gait, with their OTA values decreasing toward better symmetry in the cued condition compared to their baseline. Of these 6, 2 maintained a more symmetric OTA value in the post condition compared to their initial baseline, 3 became worse with increasing OTA values, and 1 returned back to the baseline level of asymmetry.

During the cued condition, 2 of the 11 participants became more asymmetric, with their OTA values increasing compared to their baseline. Of these two participants, one (patient code OUP05) maintained a more asymmetric OTA value in the post condition while the other (patient code MMUP03) showed a big improvement compared to the baseline.

Of the 11 participants, 3 did not show any changes in their OTA values, in either the cued or post condition. These data are summarized in Figure 7, where each level on the flowchart represents each condition (baseline, cued, and post). Temporal gait changes for each participant are color-coded: green indicates an improvement, red indicates an increase in asymmetry, and blue indicates no change. All comparisons are made against each individual's baseline values.

\section{Spatial Data}

Spatial data usefully complement temporal data for assessing changes in gait symmetry. The step length is a valuable metric for assessing the therapeutic effect of rhythm in gait rehabilitation and can be used for calculating changes in spatial gait asymmetry [24,31,32].

Two aspects of spatial data were analyzed: firstly, step length in the cued and post conditions were compared against the baseline, and secondly, the spatial asymmetry value was calculated using the same formula discussed above for the OTA.

Due to the great variability between participants, useful information can be extracted by considering both changes in mean values and changes in individual cases. Changes in mean values are considered first.

In contrast to the change in symmetry, the average stride length decreased by $16 \mathrm{~mm}$ in the cued condition compared with the baseline, and increased by almost $50 \mathrm{~mm}$ in the post condition over baseline (Figure 8). Bearing in mind that all participants in this study had experienced hemiparetic stroke with various degrees of cognitive and motor control deficiencies, it may be that the increased cognitive load of attending to the rhythm led to shorter stride lengths. A related but slightly different argument might be that asking participants to walk while following the haptic rhythm may have led to more conscious attention to their movement, increasing cognitive load and causing them to take shorter and more careful steps. Further light is cast on these hypotheses by the observation of considerably longer steps in the post condition, where the external haptic rhythm was removed and participants were asked to walk to the rhythm from memory. 
Figure 8. Average stride lengths between all participants for all three conditions: baseline, cued, and post. Stride lengths are calculated from optical marker data and analyzed using bespoke algorithms. Error bars show 1 standard error of mean.

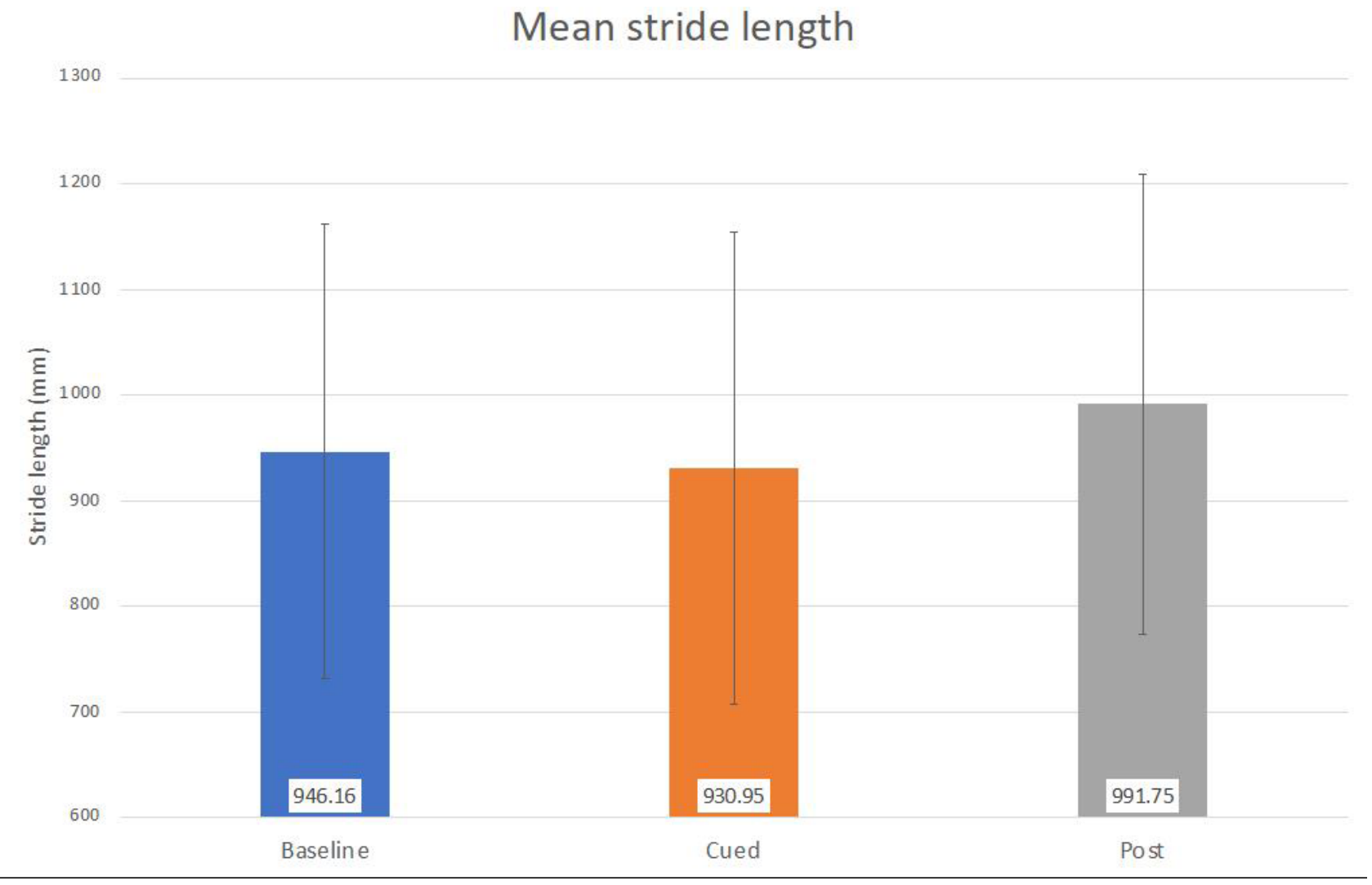

The standard error value remained similar for all conditions: SE $215 \mathrm{~mm}$, SE $223 \mathrm{~mm}$, and SE $217 \mathrm{~mm}$. This suggests that, on average, stride length did not become more or less variable during either the cued or the post condition when compared to the baseline. Unsurprisingly, in all conditions, the mean paretic stride length was shorter than the nonparetic stride length (Table 4).

Table 4. Average stride lengths of 6 participants using optical marker data from a Qualisys motion capture system. Each value represents the mean of trials in each condition (baseline, cued, post).

\begin{tabular}{lllllll}
\hline Participant code & \multicolumn{2}{l}{ Paretic leg, mean stride length $(\mathrm{mm})$} & \multicolumn{3}{c}{ Nonparetic leg, mean stride length $(\mathrm{mm})$} \\
& Baseline & Cued & Post & Baseline & Cued & Post \\
\hline MMUP01 & 1106.71 & 1086.47 & 1110.63 & 1105.03 & 1080.39 & 1108.21 \\
MMUP02 & 812.59 & 1001.08 & 1092.22 & 801.57 & 997.30 & 1090.41 \\
MMUP03 & 647.08 & 586.77 & 717.40 & 685.90 & 621.79 & 749.60 \\
MMUP04 & 1146.11 & 1182.15 & 1193.77 & 1148.95 & 1194.59 & 1208.98 \\
MMUP05 & 743.89 & 709.52 & 630.48 & 841.82 & 727.63 & 767.68 \\
MMUP06 & 1159.07 & 992.25 & 1117.81 & 1155.14 & 991.48 & 1113.84 \\
\hline
\end{tabular}

\section{Spatial Data Results Summary}

As previously noted, in situations with great individual variation, it is important to consider and analyze individual results from all participants before drawing firm conclusions. In this section, individual changes in spatial data are summarized. For 3 of the 6 participants, the mean cued stride length was shorter than the baseline. When cued, 1 participant walked with longer stride lengths, and 2 did not show any changes when walking with the cue.
When walking to the rhythm from memory in the post condition, the stride lengths of 2 participants increased, 1 decreased, and 3 showed no change. Interestingly, the same 2 participants were unchanged during cueing and post.

This data is summarized in Figure 9; each condition (baseline, cued, and post) is represented by a different level in the flowchart. Spatial changes for each participant are color-coded: green indicates an improvement, red indicates a decrease in stride lengths, and blue indicates no change. All comparisons are made against each individual's baseline values. 
Figure 9. Spatial data. The participant distribution is based on their stride-length percentage change compared to baseline. The three study conditions (baseline, cued, post) are represented by the three levels of the flowchart.

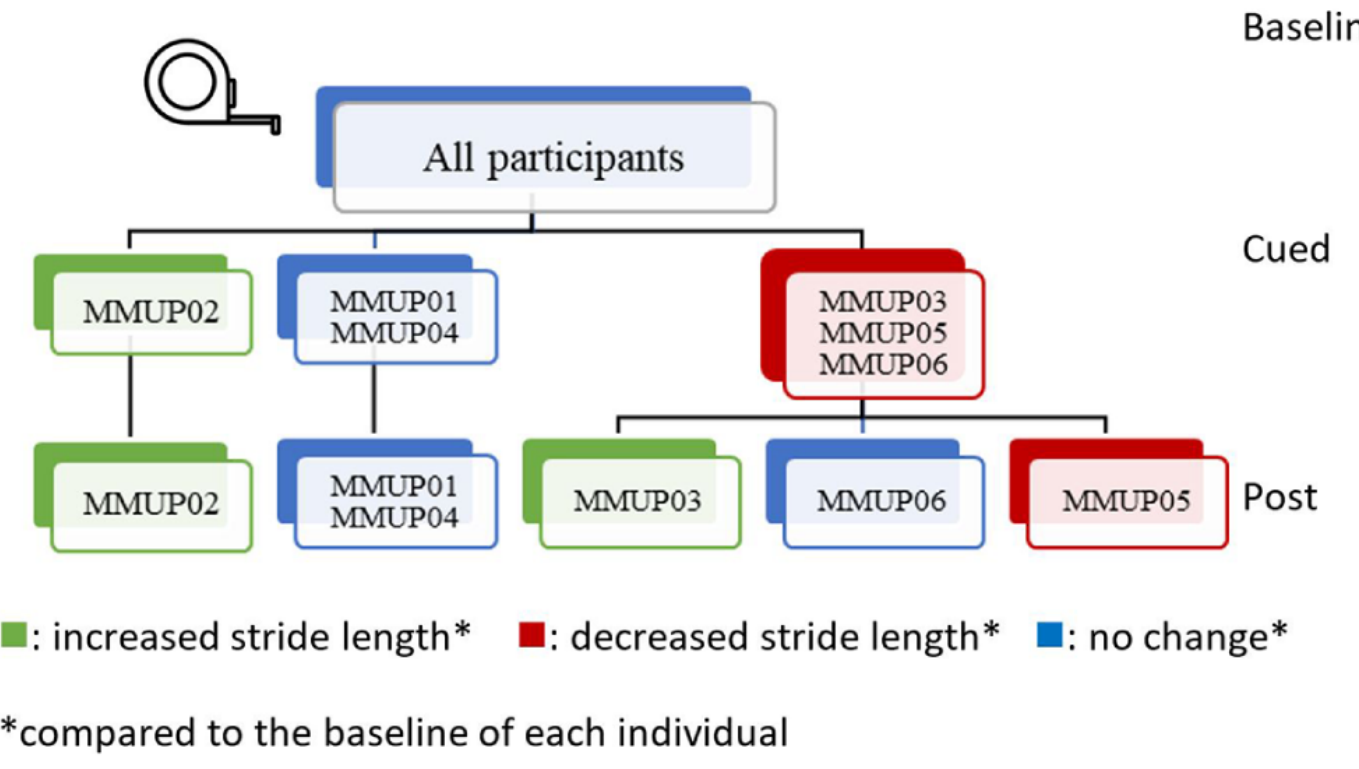

\section{Discussion}

\section{Interpretation of the Findings}

The purpose of this study was to examine the effects of rhythmic haptic cueing on spatial and temporal gait characteristics of people with hemiparesis.

Two kinds of improvement (amongst others) that physiotherapists and other relevant health professionals seek to achieve in the gait of a survivor of hemiparetic stroke are better temporal symmetry and longer stride length. One must be careful in the interpretation of quantitative information in terms of mean results from studies with a relatively small number of participants; however, we did observe overall improvements in symmetry and longer strides, both during and immediately after cueing.

Nevertheless, a more nuanced and arguably more interesting story unfolds when considering the results of individuals. Individual temporal and spatial results for each participant are presented separately in Figures 5 and 6.

\section{Temporal Walk Symmetry and Temporal Gait Pattern}

Of the 11 participants, 6 showed immediate improvements in their OTA values during the cued condition (Figure 7). This indicates a more symmetric walking pattern, with the participants spending more time on their paretic leg, reducing the burden on the nonparetic leg. Of these 6 participants, 2 retained near normative OTA value in the post condition, in which they were asked to walk to the rhythm from memory. This suggests rhythm persistence and continuing entrainment influencing their motor response after the cue is removed. An analogous phenomenon has been noted in the past by Thaut [24] where patients with hemiparesis were given an audio-rhythmic stimulus for long periods of time before asking them to walk to the rhythm from memory. Our study appears to be the first to show a similar persistence effect with a haptic cue and to demonstrate that this can take effect with relatively short exposure.
Of the 4 participants who exhibited improvements in the cued condition, 1 returned to their baseline symmetry and the other 3 showed evidence in the post condition of becoming more asymmetric than their baseline. This increase in asymmetry may be attributable to fatigue, as the post condition was at the end of the study, after the participants had already walked roughly 120 meters (2x6x10 meters). While such a distance may not tax an able-bodied person, it can be considerably taxing for people with hemiparesis. Comments from 2 participants shared that this was the longest they had walked since their stroke. Fatigue may have negatively affected the performance of other participants in the post condition without bringing it below baseline.

Moreover, 3 participants (participant codes MMUP02, OUP02, and OUP04; Figure 7) showed no change in either the cued or post conditions. This may be either because they did not understand or engage with the task or simply because they had far less room for improvement than other participants; these 3 participants had OTA values that approached normative in the baseline condition $(1.08,1.14$, and 0.79 , respectively, compared to the normative range of $0.9-1.1$ ). However, 1 of these 3 participants (participant MMUP02) showed improvement in step lengths for both conditions, compared to her baseline. This indicates the possibility of therapeutic gait-related benefits and shows the change in confidence a simple intervention can make, as well as its effect on healthier kinematics, even without a change in temporal gait pattern. Unfortunately, no spatial data were collected for the other 2 participants (participants OUP02 and OUP04).

\section{Stride Lengths Changes}

In this study, only 1 out of the 6 participants showed an immediate improvement in stride length and also maintained it during the post condition (Figure 9). Participant MMUP02, who normally uses a walking stick to ambulate, requested to do the trials without her stick, against the recommendations of one of the present physiotherapists. When asked immediately after the trials, participant MMUP02 commented, "it's when I've gone 
outside [my house] I've got no confidence, so I have the walking stick. [...] I felt a lot stronger walking in there without the stick. It made a difference in that I had a bit more confidence. I felt confident without the stick." When asked if this confidence arose from having the physiotherapist team present in the room with her, she initially agreed but then suggested further reasons: "Yes, I felt confident when she [physiotherapist] was there [next to her], but when she wasn't there, I still felt that confidence" [Participant MMUP02].

During the trials, the clinical gait analyst attending the study also commented on how the posture of this participant had changed from being crouched over her walking stick to walking upright and looking ahead. "Look how tall she [MMUP02] stands." Interestingly, participant MMUP02 is one of the participants that showed no change in her temporal data. This outcome is not entirely surprising as there is evidence in the literature to suggest that spatial and temporal parameters need not be directly related [33].

Video footage of the study was informally shown to the expert physiotherapists collaborating in this research who assisted with the initial participant recruitment for this study. As some of the study participants were their regular patients, they were also asked to comment on anything they saw as noteworthy. One of the physiotherapists remarked that participant OUP02 (who is also one of her regular physiotherapy patients) changed her habits after this study to include more walks in empty supermarket corridors during early mornings, when it is less busy. "[Participant OUP02] became more confident...(and) started using a lighter walking stick," which the therapist interpreted as another sign of increased confidence in her capabilities. While watching a video of participant OUP02 walking in one of the cued trials, the physiotherapist commented that "it's so nice to see her [OUP02] not thinking too much about her right (paretic) leg. Just let it go; let it flow."

After discussing the above findings with the physiotherapists assisting with the study, an interesting link between mood and one particular spatial (as opposed to temporal) gait characteristic was highlighted, with a potential bearing on the above results. Physiotherapists and gait rehabilitation experts routinely expect a confident mood to be associated with long strides. In contrast, in situations where hemiparetic walkers are insecure and being mindful of how they walk, shorter strides are expected. This behavior is typically observed independent of cadence (ie, walking tempo). Therefore, due to the specific link of confidence with stride lengths, the relatively short exposure to this novel approach to walking may have had a crucial role in the spatial changes observed.

Walking to a haptic rhythm mediated via a multi-limb metronome is unusual and not often encountered in everyday life. The novelty of this approach may have decreased the confidence of some participants when they walked with shorter stride lengths, as was observed in the cued condition.

However, with increased familiarity with the procedure, and having removed the task of feeling the rhythm while walking, the confidence for most participants increased and they walked with longer stride lengths (compared to their baseline) in the

post condition when asked to walk to the rhythm from memory (Figure 9).

\section{Conclusions}

This paper presents a detailed empirical study of gait rehabilitation using haptic entrainment.

This study appears to be the first systematic study of haptic cueing for gait rehabilitation. Consequently, there is not a lot of previous work available for direct comparison. However, while audio-based rhythmic cueing for rehabilitation is typically conducted differently (eg, using treadmills), previous research on it has typically used similar metrics, such as stride length and stride time [34-36], and it has been associated with a similar degree of improvement [20,36].

Given the great variability of survivors of stroke and the limited number of available participants, there is no claim here of statistical evidence able to support a formal experimental result of improved gait. However, with this participant variability in mind, this empirical gait investigation may be viewed as a set of 11 case studies and more modest empirical claims can be made. At a case study level, improvements were seen in the temporal data of 6 of the 11 study participants.

An overall beneficial decrease in the hemiparetic participants' OTA values was found, indicating an immediate improvement and a more symmetric and healthier gait pattern for 6 out of the 11 participants.

Spatial data were gathered from 6 of the 11 participants using a motion capture system; it showed 3 of the 6 participants with shorter strides with the cue than during their baseline. These spatial changes may be linked to confidence during the walk, with shorter strides indicating a temporary decrease in confidence levels or possibly a higher cognitive load. Temporal asymmetry remained improved during the post condition.

Results from the study indicate some short-term rhythm persistence in memory (post-cue condition) and entrainment to the rhythm from memory. This is not the first time this phenomenon was observed [26,27]; however, it is the first time it has been observed in the context of gait entrainment using haptically presented rhythms. This rhythm-persistence phenomenon may have significant implications for certain patients with hemiparesis who suffer from attention deficits that make it challenging to perceive the rhythm and walk at the same time. This suggests a range of studies to investigate this phenomenon in more depth in the future. This study has also shown empirically that improvements to spatiotemporal gait characteristics can occur immediately after haptic cueing, leading to a more symmetric and healthier gait pattern for a substantial portion of participants. However, this was a lab-based study, with participants with hemiparesis receiving limited exposure to the haptic rhythm. The effects of longer exposure to a haptic rhythm for gait rehabilitation in a home setting are currently unknown. A longitudinal study can be designed to investigate the effects of the haptic rhythm on patients with hemiparetic gait over longer exposure times. In addition, a longitudinal study could also include possible usability metrics (eg, increased confidence, technology acceptance) that were observed in this study but not formally investigated. 
Finally, the approach of rhythmic haptic cueing can be extended to include other neurological conditions that lead to motor and gait deficits. Literature in rhythmic auditory stimulation provides evidence of the benefits of entrainment for neurological conditions such as Parkinson disease, cerebral palsy, and
Huntington disease. An approach similar to the one adopted in this study for survivors of hemiparetic stroke and brain injury could be applied to investigate these other conditions. An initial case study with a single participant living with Huntington disease provided some encouraging results [37].

\section{Acknowledgments}

We would like to thank our participants, physiotherapists, and health professionals who unreservedly donated their time to help us in our research. Dr Theodoros Georgiou is funded by The Open University. Access to some participants and partial data for one study was assisted by funding from the Greater Manchester Academic Health Science Network. Favorable ethical approval was granted by the Open University Human Research Ethics Committee (HREC/2015/2551/Georgiou/1) and the Manchester Metropolitan University Ethics Committee (Ref: 1368). The study was carried out in accordance with the principles laid out by the Declaration of Helsinki.

\section{Conflicts of Interest}

None declared.

\section{References}

1. Mercer VS, Freburger JK, Chang SH, Purser JL. Measurement of paretic-lower-extremity loading and weight transfer after stroke. Phys Ther 2009 Jul;89(7):653-664 [FREE Full text] [doi: $\underline{10.2522 / p t j .20080230]}$ [Medline: 19465370]

2. Bohannon RW, Larkin PA. Lower extremity weight bearing under various standing conditions in independently ambulatory patients with hemiparesis. Phys Ther 1985 Sep;65(9):1323-1325. [doi: 10.1093/ptj/65.9.1323] [Medline: 4034666]

3. Min DG, Lee JH, Choe HS, Kim EJ, Shin SH, Lee JH. Comparison of bone density on the dominant and nondominant sides between healthy elderly individuals and stroke patients. J Phys Ther Sci 2016 Sep;28(9):2533-2536 [FREE Full text] [doi: 10.1589/jpts.28.2533] [Medline: 27799687]

4. Verghese J, Ambrose AF, Lipton RB, Wang C. Neurological gait abnormalities and risk of falls in older adults. J Neurol 2010 Mar 26;257(3):392-398 [FREE Full text] [doi: 10.1007/s00415-009-5332-y] [Medline: 19784714]

5. Thaut M, McIntosh G, Rice R. Rhythmic facilitation of gait training in hemiparetic stroke rehabilitation. Journal of the Neurological Sciences 1997 Oct;151(2):207-212. [doi: 10.1016/s0022-510x(97)00146-9]

6. Prassas S, Thaut M, McIntosh G, Rice R. Effect of auditory rhythmic cuing on gait kinematic parameters of stroke patients. Gait \& Posture 1997 Dec;6(3):218-223. [doi: 10.1016/s0966-6362(97)00010-6]

7. Roerdink M, Lamoth CJC, Kwakkel G, van Wieringen PCW, Beek PJ. Gait coordination after stroke: benefits of acoustically paced treadmill walking. Phys Ther 2007 Aug;87(8):1009-1022. [doi: 10.2522/ptj.20050394] [Medline: 17553922]

8. Wright RL, Masood A, MacCormac ES, Pratt D, Sackley CM, Wing AM. Metronome-Cued Stepping in Place after Hemiparetic Stroke: Comparison of a One- and Two-Tone Beat. ISRN Rehabilitation 2013;2013:1-5. [doi: $10.1155 / 2013 / 157410]$

9. Thaut M, Leins A, Rice R, Argstatter H, Kenyon G, McIntosh G, et al. Rhythmic Auditor y Stimulation Improves Gait More Than NDT/Bobath Training in Near-Ambulatory Patients Early Poststroke: A Single-Blind, Randomized Trial. Neurorehabil Neural Repair 2007 Mar 16;21(5):455-459. [doi: 10.1177/1545968307300523]

10. Roerdink M, Bank PJ, Peper CE, Beek PJ. Walking to the beat of different drums: Practical implications for the use of acoustic rhythms in gait rehabilitation. Gait \& Posture 2011 Apr;33(4):690-694. [doi: 10.1016/j.gaitpost.2011.03.001]

11. Holland S, Wright R, Wing A, Crevoisier T, Hödl O, Canelli M. A gait rehabilitation pilot study using tactile cueing following hemiparetic stroke. 2014 May Presented at: The 8th International Conference on Pervasive Computing Technologies for Healthcare; 2014; Oldenburg, Germany p. 402-405. [doi: 10.4108/icst.pervasivehealth.2014.255357]

12. Georgiou T, Holland S, van der Linden J, Tetley J, Stockley R, Donaldson G, et al. A blended user centred design study for wearable haptic gait rehabilitation following hemiparetic stroke. 2015 May Presented at: The 9th International Conference on Pervasive Computing Technologies for Healthcare (PervasiveHealth); 2015; Istanbul, Turkey.

13. Albright TD, Jessell TM, Kandel ER, Posner MI. Cell and Molecular Biology of the Neuron. In: Kandel ER, Schwartz JH, Jessell TM, Siegelbaum SA, Hudspeth AJ, editors. Principles of Neural Science. New York: McGraw-Hill; 2012.

14. Georgiou T. Rhythmic Haptic Cueing for Gait Rehabilitation of Hemiparetic Stroke and Brain Injury Survivors. Milton Keynes: The Open University; 2018.

15. Patterson KK, Parafianowicz I, Danells CJ, Closson V, Verrier MC, Staines WR, et al. Gait Asymmetry in Community-Ambulating Stroke Survivors. Archives of Physical Medicine and Rehabilitation 2008 Feb;89(2):304-310. [doi: 10.1016/j.apmr.2007.08.142]

16. Williams G. Rivermead Mobility Index. In: Kreutzer JC, DeLuca J, Caplan B, editors. Encyclopedia of Clinical Neuropsychology. New York: Springer; 2011:2186. 
17. Kobinata N, Ueno M, Imanishi Y, Yoshikawa H. Immediate effects of rhythmic auditory stimulation on gait in stroke patients in relation to the lesion site. J Phys Ther Sci 2016 Sep;28(9):2441-2444 [FREE Full text] [doi: 10.1589/jpts.28.2441] [Medline: 27799666]

18. Podsiadlo D, Richardson S. J Am Geriatr Soc 1991 Feb 27;39(2):142-148. [doi: 10.1111/j.1532-5415.1991.tb01616.x] [Medline: 1991946]

19. Bouwer A, Holland S, Dalgleish M. The Haptic Bracelets: Learning Multi-Limb Rhythm Skills from Haptic Stimuli While Reading. In: Holland S, Wilkie K, Mulholland P, Seago A, editors. Music and Human-Computer Interaction. London: Springer; 2013:101-122.

20. Thaut C, Rice R. Rhythmic auditory stimulation. In: Thaut MH, Hoemberg V, editors. Handbook of neurologic music therapy. Oxford: Oxford University Press; 2016:95-105.

21. Roerdink M, Lamoth CJC, van Kordelaar J, Elich P, Konijnenbelt M, Kwakkel G, et al. Rhythm perturbations in acoustically paced treadmill walking after stroke. Neurorehabil Neural Repair 2009 Sep 23;23(7):668-678. [doi: 10.1177/1545968309332879] [Medline: 19307435]

22. Clayton M, Sager R, Will U. In time with the music: the concept of entrainment and its significance for ethnomusicology. European Meetings in Ethnomusicology 2005;11:3-142.

23. Angelis V, Holland S, Upton PJ, Clayton M. Testing a Computational Model of Rhythm Perception Using Polyrhythmic Stimuli. Journal of New Music Research 2013 Mar;42(1):47-60. [doi: 10.1080/09298215.2012.718791]

24. Thaut MH. Neurologic Music Therapy in Sensorimotor Rehabilitation. In: Thaut MH, editor. Rhythm, Music, and the Brain: Scientific Foundations and Clinical Applications. London: Routledge; 2005:137-164.

25. Merker B. Groove or swing as distributed rhythmic consonance: introducing the groove matrix. Front Hum Neurosci 2014 Jun 23;8:454 [FREE Full text] [doi: 10.3389/fnhum.2014.00454] [Medline: 25002843]

26. Thaut MH, McIntosh KW, McIntosh GC, Hoemberg V. Auditory rhythmicity enhances movement and speech motor control in patients with Parkinson's disease. Funct Neurol 2001;16(2):163-172. [Medline: 11495422]

27. Nieuwboer A, Kwakkel G, Rochester L, Jones D, van Wegen E, Willems AM, et al. Cueing training in the home improves gait-related mobility in Parkinson's disease: the RESCUE trial. J Neurol Neurosurg Psychiatry 2007 Feb;78(2):134-140 [FREE Full text] [doi: 10.1136/jnnp.200X.097923] [Medline: 17229744]

28. Nieuwboer A. Cueing for freezing of gait in patients with Parkinson's disease: a rehabilitation perspective. Mov Disord 2008 Jul 30;23 Suppl 2(S2):S475-S481. [doi: 10.1002/mds.21978] [Medline: 18668619]

29. Holland S, Hödl O. Haptics for the Development of Fundamental Rhythm Skills, Including Multi-limb Coordination. In: Papeti S, Saitis C, editors. Musical Haptics. London: Springer; 2018:238.

30. Qualisys. Qualisys: Technical specification. Qualisys Hardware. URL: https://www.qualisys.com/hardware/ [accessed 2020-05-17]

31. Patterson KK, Gage WH, Brooks D, Black SE, McIlroy WE. Evaluation of gait symmetry after stroke: a comparison of current methods and recommendations for standardization. Gait Posture 2010 Feb;31(2):241-246. [doi:

10.1016/j.gaitpost.2009.10.014] [Medline: 19932621]

32. Thaut MH, Abiru M. Rhythmic Auditory Stimulation in Rehabilitation of Movement Disorders: A Review Of Current Research. Music Perception 2010;27(4):263-269. [doi: 10.1525/mp.2010.27.4.263]

33. Krasovsky T, Levin MF. Review: Toward a Better Understanding of Coordination in Healthy and Poststroke Gait. Neurorehabil Neural Repair 2009 Oct 12;24(3):213-224. [doi: 10.1177/1545968309348509]

34. Roerdink M, Lamoth CJC, van Kordelaar J, Elich P, Konijnenbelt M, Kwakkel G, et al. Rhythm perturbations in acoustically paced treadmill walking after stroke. Neurorehabil Neural Repair 2009 Sep;23(7):668-678. [doi: 10.1177/1545968309332879] [Medline: 19307435$]$

35. Pelton TA, Johannsen L, Huiya Chen, Wing AM. Hemiparetic stepping to the beat: asymmetric response to metronome phase shift during treadmill gait. Neurorehabil Neural Repair 2010 Jun;24(5):428-434. [doi: 10.1177/1545968309353608] [Medline: 19952366]

36. Wright RL, Bevins JW, Pratt D, Sackley CM, Wing AM. Metronome Cueing of Walking Reduces Gait Variability after a Cerebellar Stroke. Front Neurol 2016 Jun 01;7:84 [FREE Full text] [doi: 10.3389/fneur.2016.00084] [Medline: 27313563]

37. Georgiou T, Islam R, Holland S, van der Linden J, Price B, Mulholland P, et al. Rhythmic Haptic Cueing Using Wearable Devices as Physiotherapy for Huntington Disease: Case Study. JMIR Rehabil Assist Technol 2020 Sep 14;7(2):e18589 [FREE Full text] [doi: 10.2196/18589] [Medline: $\underline{\text { 32924955] }}$

\section{Abbreviations}

IMU: inertia monitoring unit

OTA: overall temporal asymmetry

RAS: rhythmic auditory stimulation

RMA: Rivermead Motor Assessment 
Edited by G Eysenbach; submitted 10.03.20; peer-reviewed by L Shum; comments to author 27.04.20; revised version received 19.06.20; accepted 11.11.20; published 24.11.20

Please cite as:

Georgiou T, Holland S, van der Linden J

Rhythmic Haptic Cueing for Gait Rehabilitation of People With Hemiparesis: Quantitative Gait Study

JMIR Biomed Eng 2020;5(1):e18649

URL: http://biomedeng.jmir.org/2020/1/e18649/

doi: $10.2196 / 18649$

PMID:

(C)Theodoros Georgiou, Simon Holland, Janet van der Linden. Originally published in JMIR Biomedical Engineering (http://biomedeng.jmir.org), 24.11.2020. This is an open-access article distributed under the terms of the Creative Commons Attribution License (https://creativecommons.org/licenses/by/4.0/), which permits unrestricted use, distribution, and reproduction in any medium, provided the original work, first published in JMIR Biomedical Engineering, is properly cited. The complete bibliographic information, a link to the original publication on http://biomedeng.jmir.org/, as well as this copyright and license information must be included. 